\title{
Novel immunocontraceptive targets in mammals: uterine secretions and the conceptus; a marsupial approach
}

\author{
E M Menkhorst, S Cui and L Selwood \\ Department of Zoology, The University of Melbourne, Parkville, Victoria 3010, Australia \\ Correspondence should be addressed to L Selwood; Email: I.selwood@zoology.unimelb.edu.au \\ E M Menkhorst is now at Prince Henry's Institute of Medical Research, Clayton, Victoria 3168, Australia
}

\begin{abstract}
We report the first immunocontraceptive trial in mammals using a uterine-secreted protein, the marsupial shell coat protein 4 (CP4). The marsupial shell coat, which surrounds the conceptus for $60-80 \%$ of gestation, is secreted by the uterine epithelium. Following immunization against glutathione S-transferase (GST)-CP4, the fertility of female common brushtail possums $(n=6)$ was significantly reduced $(P=0.000)$, and this reduction in fertility was positively correlated with the maximum GST-CP4 humoral immune response $(P=0.025)$. Ultrastructural examination of the reproductive tract indicated that the cell-mediated immune response against GST-CP4 targeted the shell coat, the shell-free conceptus and the uterine glandular epithelium, thus preventing normal conceptus development and uterine secretion of shell coat proteins and nutrients. These results show that uterine-secreted proteins are promising immunocontraceptive targets, especially in pest mammal species, e.g. possum, rabbit and horse, that have uterine-secreted additions to embryonic coats, or that have late implantation requiring uterine nutrient provisioning from secretions.

Reproduction (2008) 136 471-480
\end{abstract}

\section{Introduction}

This study reports the first in vivo investigation into the use of uterine-secreted proteins as immunocontraceptive targets in mammals. The uterus forms part of the common mucosal immune system, which is potentially in contact with the external environment. Except for highly organized secondary lymphoid nodules, the mammalian uterus contains the full range of lymphohaemopoietic cells and molecular regulators required for 'adaptive' (antigen-specific) immunity (Robertson 2000).

The immune response of the eutherian uterine endometrium is likely to be T-cell mediated (Robertson 2000). Major histocompatibility class (MHC) $\mathrm{II}^{+}$dendritic cells, macrophages and lymphocytes form a contiguous layer beneath the epithelial surface in the eutherian endometrium and are considered the major antigen-presenting cells (APC; Robertson 2000). In the rat, uterine epithelial cells express $\mathrm{MHC} \mathrm{II}$ molecules that stimulate T-cells to release cytokines in vitro (Wira \& Rossoll 1995), suggesting that they may play a surveillance role (Quayle 2002).

The dichotomy between the uterus mounting a rapid response to bacteria and the apparent suppression of an immune response directed against sperm and the conceptus is not fully understood (Quayle 2002). However, in eutherians, semen, the conceptus (Robertson 2000), progesterone (Piccinni 2003, Lewis 2004) and in mice, early pregnancy factor (EPF; Morton 1998) induce the expression of specific cytokines able to skew the differentiation and expansion of certain lymphocyte populations, suppressing the immune response. Further immunization during oestrus results in a lower immunoglobulin response than at other times in both rats (Wira \& Rossoll 1995) and mice (Gockel et al. 2000).

Little is known of the marsupial uterine immune response. In an oestrous cycle-dependent manner, a layer of macrophages and lymphocytes, presumably capable of acting as APCs, is found beneath the luminal epithelium in didelphid and dasyurid marsupials (Padykula 1976, Cruz \& Selwood 1993, 1997, Wick \& Kress 2002) and EPF has been identified in some marsupials (Cruz et al. 2006), including Trichosurus vulpecula (Cui et al. 2001).

The common brushtail possum, T. vulpecula, is the major ecological and economic vertebrate pest species in New Zealand. Immunocontraception targeting the marsupial shell coat, which is secreted by the uterine epithelium and surrounds the conceptus for $60-80 \%$ of gestation (Selwood 2000), is a potential fertility control method. Cleavage will proceed without a shell coat in vitro (Moore \& Taggart 1993, Renfree \& Lewis 1996, Casey \& Selwood 2003), but shell-free conceptuses do not form normal blastocysts. Removal of the shell coat from T. vulpecula 32-cell and later stage blastocysts results in compromised development, with cells 
becoming rounded and forming a highly abnormal epithelia (Casey \& Selwood 2003), suggesting that at these stages of development, the presence of the shell coat preserves blastocyst epithelial integrity.

Coat protein 4 (CP4), a marsupial shell coat protein identified in $T$. vulpecula, is the first uterine-secreted embryonic coat protein identified in a mammal (Casey et al. 2002). CP4 is a unique protein; however, the $\mathrm{N}$-terminus of the amino acid sequence shares significant homology with the C-terminus of $\alpha$-enolase (Cui \& Selwood 2003), a glycolytic enzyme, expressed in a wide variety of tissues, including muscle and stimulated immune cells (Pancholi 2001). The $\alpha$-enolase gene also codes for $\tau$-crystallin (Wistow et al. 1988), a structural lens protein found in lampreys, some fish, reptiles, birds (Wistow et al. 1988) and, according to one study, mammals, including the marsupial Didelphis virginiana but not Macropus eugenii (Wistow \& Kim 1991). $\tau$-Crystallin has low enzymatic activity due to posttranslational monomerization (Wistow et al. 1988). CP4 is not predicted to be enzymatic, as the enzymatic domain region of the $\alpha$-enolase amino acid sequence, indicated by the presence of glutamines, is not homologous with CP4 (Cui \& Selwood 2003). CP4 is thought to be a structural protein in the marsupial shell coat (Cui \& Selwood 2003), as $\tau$-crystallin is a structural lens protein, and the vertebrate lens and marsupial shell coat share similar properties: they are both translucent, with significant ability to modulate their form (Cui \& Selwood 2003) and have little turnover once formed (Wistow \& Piatigorsky 1988, Piatigorsky 1989, Cui \& Selwood 2003).

It is anticipated that immunization against CP4 will (1) interfere with shell coat formation and maintenance, thereby preventing normal blastocyst development and ending the pregnancy and (2) result in an immune response targeting the uterine glandular epithelium, which secretes the shell coat and other proteins required during pre-implantation development. Here, we present results from the first mammalian immunocontraceptive trial against a uterine-secreted protein. We show the effect of immunization against glutathione S-transferase (GST)-CP4 on the humoral immune response, fertility, and uterine and conceptus morphology. Also, for the first time, the cell population responsible for secreting a marsupial shell coat protein, CP4, is identified, and the pattern of $\mathrm{CP} 4$ expression by the uterine epithelium during gestation is presented.

\section{Results \\ CP4 expression in the uterus during the oestrous cycle}

Immunohistochemistry against GST-CP4 showed that CP4 was expressed by the luminal and glandular uterine epithelia in T. vulpecula (Fig. 1A-L). During the oestrous cycle, expression by the glandular epithelium, as evidenced by secretory product in the glandular lumen and cytoplasm of glandular epithelial cells, was biphasic, beginning just prior to ovulation (Fig. 1A) and continuing during cleavage stages (Fig. 1B) but was not evident in unilaminar (Fig. 1C) and bilaminar (not shown) blastocyst stages, in which staining was also present in the negative control (Fig. 1I). CP4 expression occurred again, mainly in the apical glandular epithelium, during the trilaminar blastocyst stage (Fig. 1D and E), and continued only in the apical glandular epithelium during the embryonic (not shown) and fetal (Fig. 1F) stages of development. During cleavage stages (Fig. 1B), CP4 expression was patchy between the cells of the apical glandular epithelium but was more constant basally. The expression of $\mathrm{CP} 4$ by the uterine luminal epithelium was not biphasic. Luminal CP4 expression began during cleavage (Fig. 1B inset), and continued until the trilaminar blastocyst stage (Fig. 1D). Positive staining of the luminal epithelium found during the embryo and fetus stages of development did not appear to be a result of secretory activity (Fig. $1 \mathrm{~F}$ inset), and may reflect staining of $\alpha$-enolase, a glycolytic enzyme with which CP4 shares significant homology at the $N$-terminus (Cui \& Selwood 2003). $\alpha$-Enolase is expressed by muscle and activated immune cells in eutherians (Pancholi 2001) and in T. vulpecula the GSTCP4 antibody identified stromal lymphocytes and distinct cell populations in the myometrium (not shown).

\section{Immune response}

A sera immune response against GST-CP4 was detected by ELISA in all immunized animals. The average GSTCP4 immune titre reached a maximum 2 months after the initial immunization (Fig. 2A), before decreasing to pre-immunization levels 9-10 months after the initial immunization (Fig. 2A).

\section{Fertility}

Prior to GST-CP4 immunization, $0.90 \pm 0.05(n=6)$ of pre-immunization oestrous cycles, identified by daily urine sampling (Duckworth et al. 1998b), resulted in a birth. Following immunization against GST-CP4, births occurred in only $0.55 \pm 0.07(n=6)$ of oestrous cycles (Table $1 ; P=0.000$ ). The duration of reduced fertility varied between 8 and 38 months (Table 2), with a mean of 16.5 months $( \pm 5.2)$. The proportion of fertile oestrous cycles was not associated with the immune titre at the time of oestrous cycling $(n=57$; Fig. 2B; $P=0.625)$. The percentage reduction in fertility following immunization was positively correlated with the maximum GST-CP4 immune titre recorded (Fig. 2C; $P=0.025$ ). A third booster injection of GSTCP4 was given to three of the six females at the end of the luteal phase; however, this did not result in a further reduction in fertility (Table 1 ). 

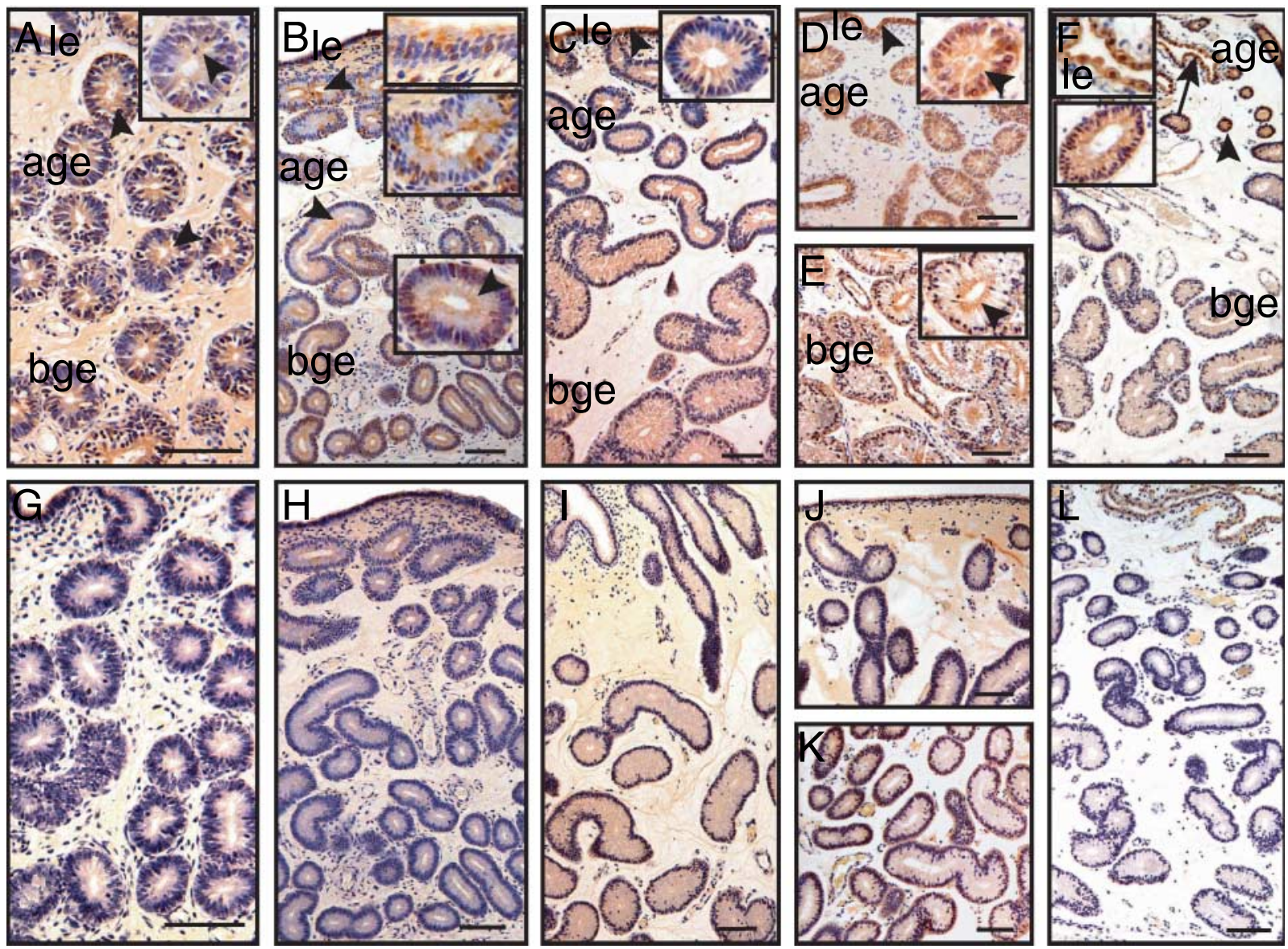

Figure 1 Trichosurus vulpecula uterine endometrium showing CP4 expression by the uterine epithelium as identified by anti-GST-CP4 mouse antisera. (A) Late pre-ovulatory ( $4 \mathrm{~mm}$ follicle) stage endometrium showing CP4 expression (arrowheads) in the luminal surface of the glandular epithelium and in the lumen. Inset shows a representative section from the apical glandular epithelium (age), magnified $1.5 \times$ greater than the basic figure. (B) Cleavage stage endometrium showing expression of CP4 on the apical surface of the glandular epithelium (arrowheads). CP4 expression was strong in individual cells of the luminal (le) and apical glandular epithelium (insets), but weak in the basal glandular epithelium (bge; inset). Insets show a representative section from the luminal, apical and basal glandular epithelium, magnified $3 \times$ greater than the basic figure.

(C) Unilaminar blastocyst stage endometrium showing CP4 expression (arrowhead) in the luminal epithelium, but reduced expression in the apical and basal glandular epithelium. Inset shows representative gland from the apical glandular epithelium, magnified $2.5 \times$ greater than the basic figure. (D and E) Trilaminar blastocyst stage endometrium. Strong CP4 expression (arrowheads) was found in the (D) luminal, apical and (E) basal glandular epithelium. Inset shows a representative gland from the apical and basal glandular epithelium, showing intense staining in the cytoplasm, magnified $2.5 \times$ greater than the basic figure. (F) Fetus-stage endometrium showing strong CP4 expression in the apical glandular epithelium (arrowhead).

Strong staining was also observed in the luminal epithelium (arrow), but cells of the luminal epithelium did not appear secretory (inset). Insets show representative areas of the luminal and apical glandular epithelium, magnified $2.5 \times$ greater than the basic figure. (G-L) Equivalent sections to (A-F) incubated with pre-immunization IgG control antisera. All sections were stained with DAB and haematoxylin. Scale bars represent $100 \mu \mathrm{m}$.

\section{Tissue response}

Except for the uterus and conceptus, histology of all tissues collected from GST-CP4-immunized animals showed normal morphology (data not shown) when compared to GST and PBS immunized T. vulpecula. The ovaries of all females indicated recent oestrous cycling, with corpora lutea, corpora albicans and Graafian follicles visible (data not shown).

\section{Uterus}

All six females immunized against GST-CP4 showed varying degrees of a uterine immune response directed against the glandular epithelium. Initially, when the immune response was slight, some glandular epithelial cells became necrotic and detached from the epithelium. In a more advanced immune response, lymphocytes were observed adhering to the necrotic glandular epithelium that had been removed in strips from the basal lamina (Fig. 3A). Lymphocytes accumulated in the glandular lumen, and in some cases apparently blocked the exit of the gland into the uterine lumen, because the cellular material was clumped and the glandular lumen became extended (Fig. 3B).

The loss of a large amount of the glandular epithelium led to a significant reduction in endometrial width when compared with published normal endometrial widths 

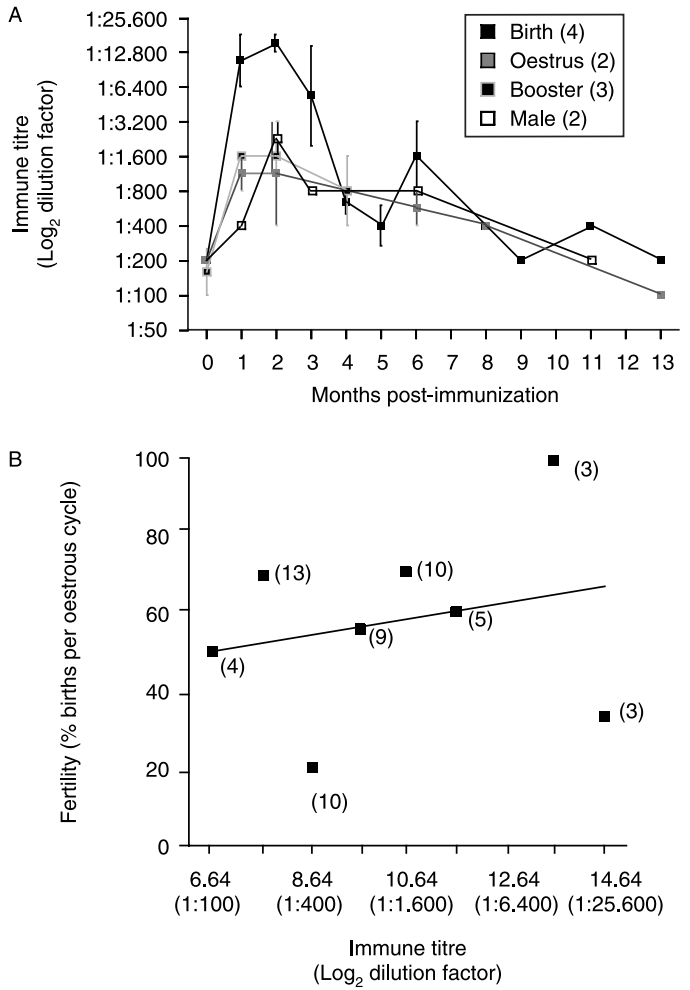

C

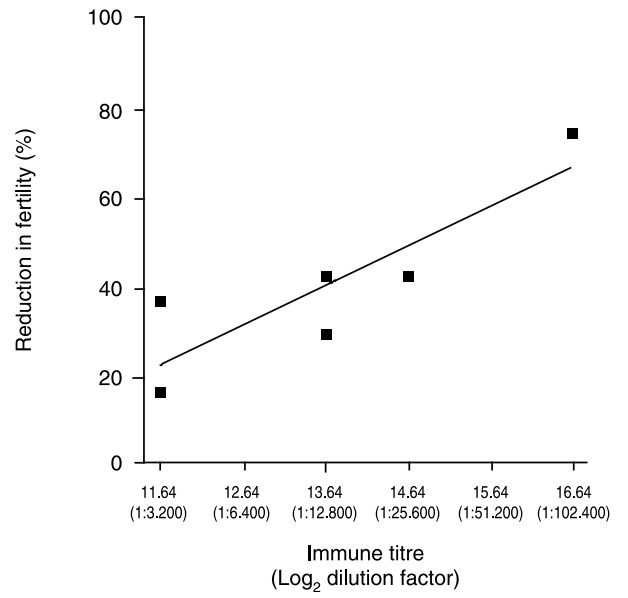

Figure 2 (A) The average immune titre over time (months) in Trichosurus vulpecula immunized against GST-CP4 around the time of birth (black, $n=4$ ), during oestrus (grey, $n=2$ ), males (white, $n=3$ ) and those females given a third booster (black/grey, $n=3$ ). Time 0 is the time of blood sampling just prior to the initial immunization in females immunized around birth, oestrus and males, but is the time of sampling just prior to the booster immunization in females given a third booster. The immune titre peaked 2 months after initial immunization and it had fallen to preimmune levels 9-10 months after the initial immunization. (B) The percentage of oestrous cycles that included a birth in female $T$. vulpecula following immunization against GST-CP4 was not correlated with the GST-CP4 immune titre found during that oestrous cycle $(r=0.206, n=8$, $P=0.625)$. The number of cycles analysed for each immune titre is given in parentheses. (C) The total reduction in fertility $(\%)$ in female T. vulpecula following immunization against GST-CP4 was positively correlated ( $r=0.867, n=6, P=0.025$ ) with the maximum GST-CP4 immune titre. The actual dilution factor is given in brackets beneath the $\log _{2^{-}}$ transformed immune titre, on the $x$-axis in (B) and (C).
(Shorey \& Hughes 1973). In all GST-CP4 immunized females, the width of the ipsilateral $(1.67 \pm 0.52 \mathrm{~mm}$, $n=6 ; t=2.570$, df $5, P=0.050)$ and contralateral $(1.47 \pm 0.31 \mathrm{~mm}, n=6 ; t=4.899$, df $5, P=0.004)$ uterine endometrium was thinner and contained little secretory material (Fig. 3C) when compared with the published normal endometrium width, $3 \mathrm{~mm}$ (Shorey \& Hughes 1973; Fig. 3D).

Ultrastructural studies confirmed that the remaining glandular epithelium in GST-CP4-immunized animals contained mainly undifferentiated, non-secretory epithelial cells (Fig. 4A), together with ciliated cells and monocytes (Fig. 4A). The multilayered complex of stromal cells that surrounded the glandular epithelium in the GSTCP4 immunized endometrium (Fig. 3A and B) included mast cells (Fig. 4A) and lymphocytes (data not shown).

Immune cells found in the glandular lumen included actively phagocytosing macrophages (Fig. 4B) and lymphocytes (Figs $3 \mathrm{~A}$ and $\mathrm{B}$ and $4 \mathrm{C}$ ), which were occasionally found adhering to secretory glandular epithelial cells (Fig. 4C), that, in this case, had detached from the glandular epithelium, suggesting that secretory cells of the glandular epithelium were recognized as the source of CP4.

\section{Conceptus}

The immune response also targeted embryonic contents in the uterine lumen. Of the four females killed postovulation, two had degraded conceptuses without a shell coat (Fig. 5A) and two were surrounded by an apparently normal shell coat (Fig. 5B) but with mucoid coat irregularities (Fig. 5B).

In the shell-free conceptuses, neutrophils were found actively phagocytosing the degraded conceptus cytoplasm and mucoid coat (Fig. 5C). The contents of both shell-free conceptuses were significantly degraded, with no obvious polarity. The mucoid coat contained remnants of sperm tails (Fig. 5D) and the degraded conceptuses were confirmed as being embryonic in origin by the presence of filamentous mitochondria (Fig. 5E) showing the radial external filaments and internal cristae that characterize the $T$. vulpecula zygote (Frankenberg \& Selwood 1998). Also visible was electron-dense granular material (Fig. 5F), degraded vesicles (Fig. 5G) and microvilli (Fig. 5H), all of which are found during the zygote and early cleavage stages of T. vulpecula (Frankenberg \& Selwood 1998).

\section{Discussion}

This study illustrates for the first time in vivo the potential of uterine-secreted proteins as immunocontraceptive targets in mammals. The cell-mediated immune response generated against GST-CP4 attacked not only the shell coat but also the glandular epithelium, which should prevent secretion of the shell coat and essential 
Table 1 The proportion, pre- and post-glutathione S-transferase-coat protein 4 (GST-CP4) immunization, of oestrous cycles in which a birth occurred, the percentage reduction in fertility following immunization and the maximum immune titre against GST-CP4 for each female Trichosurus vulpecula immunized.

Births per oestrous cycle

\begin{tabular}{|c|c|c|c|c|}
\hline Female $T$. vulpecula & Pre-immunization (cycles) & Post-immunization (cycles) & Reduction in fertility (\%) & $\begin{array}{l}\text { Maximum immune titre } \\
\text { against GST-CP4 } \\
\text { (log } \log _{2} \text { dilution factor) }\end{array}$ \\
\hline $13 \mathrm{O}$ & $0.94(16)$ & 0.60 & 36.2 & $1: 3200(11.64)$ \\
\hline $19 \mathrm{O}^{+}$ & $0.75(4)$ & $0.63(19)$ & 16.0 & $1: 1600(10.64)$ \\
\hline $25 \mathrm{~L}^{+} \mathrm{P}$ & $1.00(3)$ & $0.58(11)$ & 42.0 & $1: 25600(14.64)$ \\
\hline $28 \mathrm{~L}^{+} \mathrm{P}$ & $1.00(3)$ & $0.58(12)$ & 42.0 & $1: 12800(13.64)$ \\
\hline $10 \mathrm{LP}$ & $0.73(11)$ & $0.19(27)$ & 73.9 & $1: 102400(16.64)$ \\
\hline $24 \mathrm{~L} \mathrm{~N}$ & $1.00(1)$ & $0.71(14)$ & 29.0 & 1:12 800 (13.64) \\
\hline Mean \pm S.E.M. & $\begin{array}{l}0.90 \pm 0.05(38) \\
\chi^{2}=14.446, \mathrm{df} 1 \\
P=0.000^{\mathrm{a}}\end{array}$ & $0.55 \pm 0.07(88)$ & $\begin{array}{l}39.9 \pm 7.9 \\
r=0.867, \text { df } 6 \\
P=0.025^{b}\end{array}$ & $1: 12800(13.64)$ \\
\hline
\end{tabular}

${ }^{+}$Indicates a third booster given at 18,9 and 10 months following the initial immunization respectively. S.E.M., standard error of the mean; L, immunized during the late luteal phase; $\mathrm{N}$, non-pregnant at the time of immunization; $\mathrm{O}$, immunized during oestrus; $\mathrm{P}$, pregnant at the time of immunization. ${ }^{a}$ Indicates a significant difference in fertility between the pre- and post-immunization periods. ${ }^{\mathrm{b}}$ Indicates a significant correlation between the percentage reduction in fertility and the maximum immune titre against GST-CP4 observed.

nutrients during the pre-implantation period. This vaccine, therefore, has a double mechanism to interfere with fertility and opens up an array of potential immunocontraceptive targets for a wide variety of mammals, including pest marsupial populations requiring fertility control such as possums, koalas and eastern grey kangaroos. Fertility control in pest eutherian mammals that have uterine contributions to the egg and embryonic coats, or those that have a long preimplantation period requiring nutrient provisioning by the uterus, such as the rabbit (Denker \& Gerdes 1979) and the horse (Herrler et al. 1998), is also possible using uterine-secreted targets.

The continued expression of CP4 during the embryo and fetus stages in T. vulpecula, shown for the first time here, provides strong evidence that a shell coat is essential for marsupial conceptus development during all of the pre-implantation period. The second wave of expression, which corresponds with the previously identified second wave of cp4 mRNA expression (Cui \& Selwood 2003), began during the trilaminar blastocyst stage, a period of rapid conceptus expansion, suggesting that a minimum thickness of shell coat is required during the pre-implantation period.

The humoral immune response generated in this study was more similar to a eutherian primary humoral immune response, with a high initial response falling away to preimmune levels (Fig. 2A), than previously shown in most marsupial studies, where the initial response is slower (Rowlands 1970) and lower (Taylor \& Burrell 1968, Doolin et al. 2002), but once initiated remains high for a much longer period (Wilkinson et al. 1994, Duckworth et al. 1998a, Kitchener et al. 2002, Kay \& Kitchener 2003), as long as 37 weeks in Monodelphis domestica (Croix et al. 1989). The results of this study support Kitchener et al. (2002) who also did not find a slow or low marsupial primary humoral immune response, but the immune titre in that study remained high for a longer period than found here. The secondary immune response after the third booster immunization shown here (Fig. 2B), in $M$. domestica (Croix et al. 1989) and in M. eugenii (Kitchener et al. 2002) was weaker than in eutherian models.

Table 2 The humoral and tissue responses of female Trichosurus vulpecula immunized against glutathione S-transferase-coat protein 4 (GST-CP4) associated with age, timing of initial immunization and the timing of tissue collection.

\begin{tabular}{|c|c|c|c|c|c|c|c|c|}
\hline \multirow[b]{2}{*}{$\begin{array}{l}\text { Female } \\
\text { T. vulpecula } \\
\text { number }\end{array}$} & \multirow[b]{2}{*}{$\begin{array}{l}\text { Hierarchy of } \\
\text { estimated age } \\
\text { at initial } \\
\text { immunization }\end{array}$} & \multirow[b]{2}{*}{$\begin{array}{l}\text { Maximum } \\
\text { immune titre }\end{array}$} & \multirow[b]{2}{*}{$\begin{array}{l}\text { Reduction } \\
\text { in fertility }\end{array}$} & \multirow[b]{2}{*}{$\begin{array}{l}\text { Duration of } \\
\text { reduced fertility } \\
\text { (months) }\end{array}$} & \multirow[b]{2}{*}{$\begin{array}{l}\text { Initial } \\
\text { immunization } \\
\text { given during }\end{array}$} & \multirow[b]{2}{*}{$\begin{array}{l}\text { Uterine } \\
\text { response }\end{array}$} & \multicolumn{2}{|c|}{ Time of tissue collection } \\
\hline & & & & & & & $\begin{array}{l}\text { Stage of } \\
\text { oestrous } \\
\text { cycle }\end{array}$ & $\begin{array}{l}\text { Months after initial } \\
\text { immunization }\end{array}$ \\
\hline 13 & 1 (oldest) & 5 & 4 & 8 & Oestrus & + & Pre-ov & 8 \\
\hline 10 & 2 & 1 (highest) & 1 (highest) & 38 & Late luteal & +++ & Post-ov & 39 \\
\hline $19^{a}$ & 3 & 6 (lowest) & 6 (lowest) & 26 & Oestrus & +++ & Post-ov & 26 \\
\hline $25^{\mathrm{a}}$ & 4 & 2 & 3 & 10 & Late luteal & ++ & Post-ov & 14 \\
\hline $28^{\mathrm{a}}$ & 5 & 4 & 3 & 9 & Late luteal & + & Pre-ov & 13 \\
\hline 24 & 6 (youngest) & 4 & 5 & 8 & Late luteal & ++ & Post-ov & 16 \\
\hline
\end{tabular}

Uterine immune response: +++ , advanced; ++ , medium; + , slight. Pre-ov, pre-ovulation; Post-ov, post-ovulation.

${ }^{\mathrm{a}}$ Third booster immunization given. 

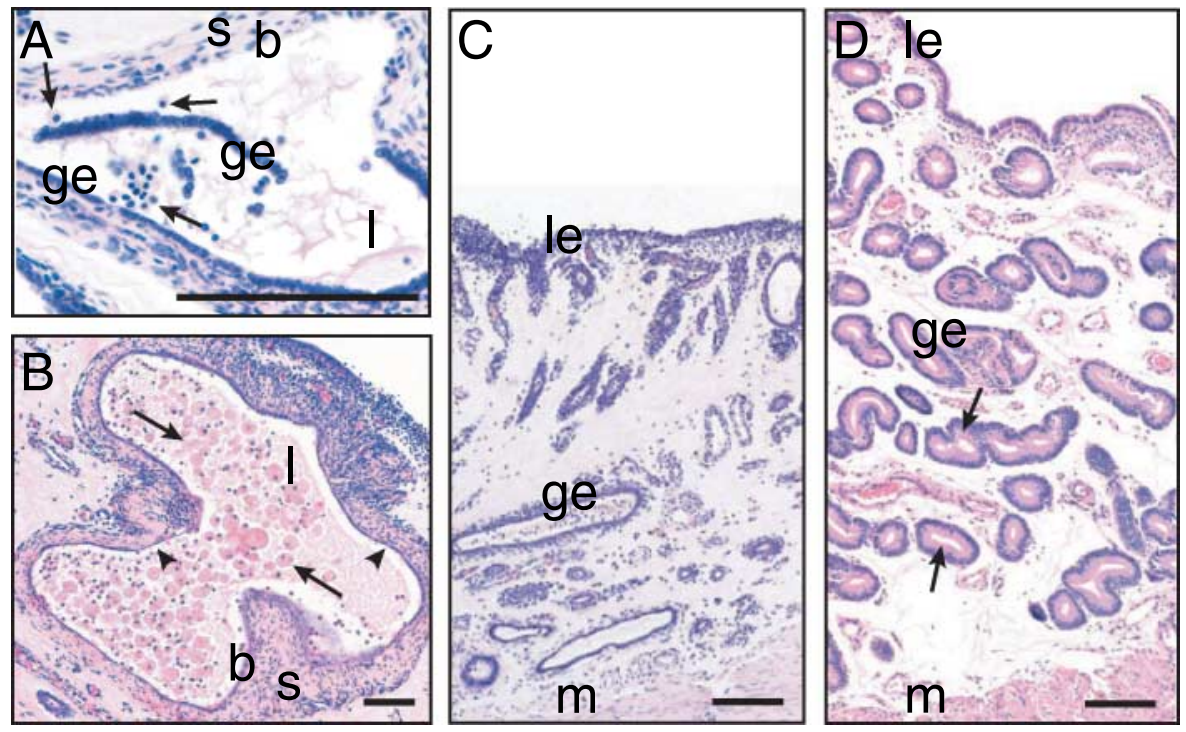

Figure 3 Histological sections of the uterine endometrium in female Trichosurus vulpecula immunized against GST-CP4 showing: (A) Lymphocytes (arrows) in the glandular lumen (I) associated with necrotic glandular epithelial cells (ge) removed from the level of the basal lamina (b). The base of the gland was surrounded by a multilayered complex of stromal cells (s). (B) An accumulation of lymphocytes (arrows) in the swollen glandular lumen. The level of the basal lamina (b) was surrounded by stromal cells (s) but glandular epithelial cells (arrowheads) contained little secretory material. (C) The uterine endometrium on day 1 after ovulation was much thinner with fewer glands (ge), containing little secretory material, than a normal endometrium from unimmunized animals (D). (D) The normal uterine endometrium from an unimmunized animal on day 1 after ovulation, contained secretory material (arrows) in the well-developed glandular epithelium (ge). This section represents a relatively thin region of the normal endometrium on day 1 , to allow its illustration at a similar scale to the immunized endometrium. le, luminal epithelium; m, myometrium. Scale bars represent $100 \mu \mathrm{m}$.

The clustering of stromal immune cells around the targeted glandular epithelium suggests that the uterine immune response in T. vulpecula was cell mediated, as occurs in eutherians (Robertson 2000, Piccinni 2003). The ability of serum immune titres (IgG) generated against $\mathrm{CP} 4$ to predict the duration of the contraceptive uterine immune response was limited. The rapid return of serum immune titres to pre-immune levels following both primary and secondary immunizations may possibly result because all subsequent antigen presentation occurred in the uterine lumen. In future studies, CP4 should be conjugated with an immunogenic protein, such as keyhole limpet haemocyanin, to increase immunogenicity and the cell-mediated immune response.

Neutrophils, which are involved in the initial attack against bacteria entering the uterus (Targowski 1984, Lewis 2004), attacked the shell-free conceptus, providing the first experimental evidence that the marsupial shell coat provides an immunoprotective barrier between the conceptus and the maternal immune system. Although shell-free conceptuses are able to survive in vitro during cleavage (Moore \& Taggart 1993, Renfree \& Lewis 1996, Casey \& Selwood 2003), the presence of neutrophils attacking the shell-free conceptus in vivo shown here and in vitro conceptus failure during blastocyst formation after shell removal (Casey \& Selwood 2003), show that the long-term survival of a conceptus would be compromised in GST-CP4-immunized animals.

Surprisingly, only the uterus showed an adverse response to immunization against CP4 (data not shown) despite (1) the sequence homology between CP4 and $\alpha$-enolase and (2) the fact that cp 4 transcription has been identified in the heart, liver, spleen and oviduct as well as the uterus in T. vulpecula (Cui \& Selwood 2003). This suggests that (1) the immune response in $T$. vulpecula recognized epitopes from the novel, C-terminus, region of $\mathrm{CP} 4$ and not the region homologous with $\alpha$-enolase and (2) CP4 may not be translated, or at least not secreted, by tissues other than the uterus, therefore, these cells were not recognized by the immune response generated against CP4. Further investigation into the role and expression of $\mathrm{CP} 4$ in these tissues is required to understand the lack of immune response observed in this study.

This study has demonstrated the potential of uterinesecreted proteins as immunocontraceptive targets for any mammal, including humans, as immunization against GST-CP4 resulted not only in an immune response directed against the coat and conceptus but also against the uterine glandular epithelium itself, preventing the secretion of essential nutrients to the conceptus during the pre-implantation period, and possibly affecting the ability of the uterus to respond to implantation signals. 

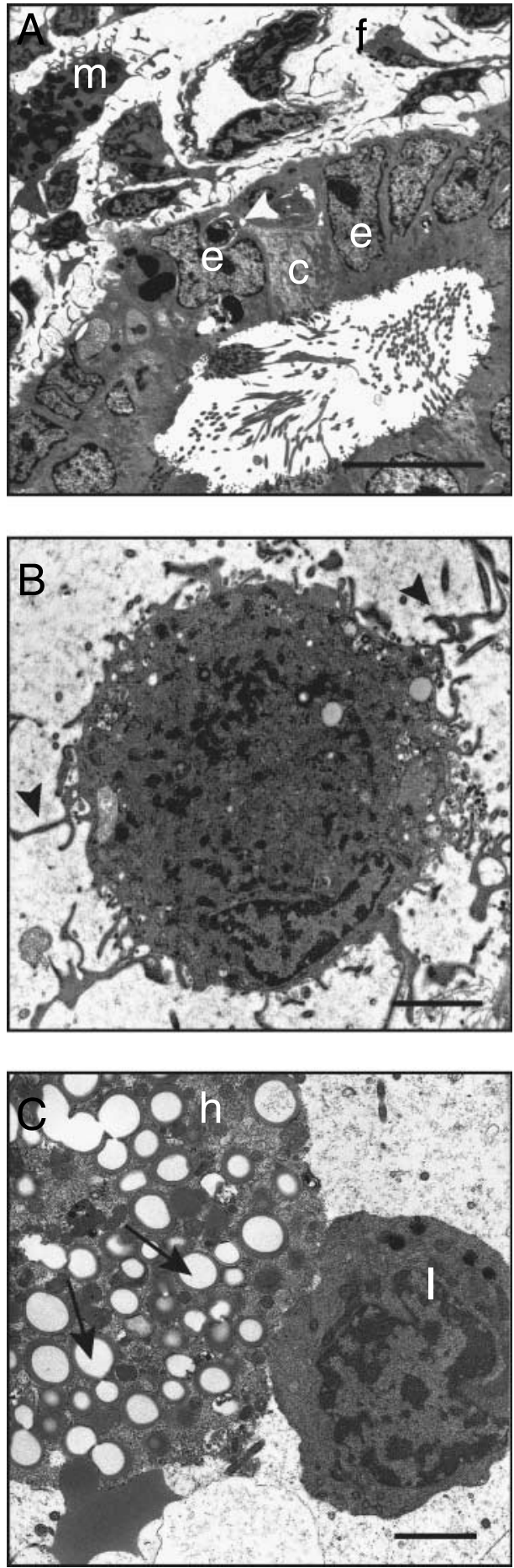

Figure 4 Electron micrographs of the uterus in female Trichosurus vulpecula immunized against GST-CP4 showing: (A) The glandular epithelium contained undifferentiated epithelial cells (e), ciliated cells (c) and monocytes (arrowheads). Surrounding the glandular epithelium in the stroma were mast cells $(\mathrm{m})$ and fibroblasts ( $\mathrm{f}$ ). Scale bar represents $10 \mu \mathrm{m}$. (B) Actively phagocytosing macrophages with long pseudopodia (arrowheads) were found in the glandular lumen. Scale bar represents $2 \mu \mathrm{m}$. (C) Lymphocytes (I), shown here adhered to a holocrine secretory glandular epithelial cell (h), which contained numerous secretory vesicles (arrow), were found in the glandular lumen. Scale bar represents $2 \mu \mathrm{m}$.

\section{Materials and Methods}

\section{Animals}

Wild-trapped T. vulpecula were trapped in New Zealand under permits from The Ministry of Agriculture and Fisheries, New Zealand. The colony of $T$. vulpecula used as experimental animals for this study operated under permits from the Victorian Government Department of Sustainability and Environment and The University of Melbourne Animal Ethics Committee, and followed conventions of the Australian National Health and Medical Research Council 'Australian Code of Practice for the Care and Use of Animals for Scientific Purposes'.

\section{Recombinant GST-CP4 production}

The T. vulpecula cp4 cDNA sequence (Cui \& Selwood 2003) was introduced into a bacterial expression system, the pGEX2T plasmid expression vector (Pharmacia), which was transformed into competent Escherichia coli (JM109; Promega). The expression and purification of recombinant GSTCP4 was as previously described for GST-vehicle associated protein (VAP) 1 expression in Cui et al. (2005).

\section{Immunohistochemistry}

T. vulpecula uterine samples were obtained from wild-trapped animals in New Zealand between 1995 and 2000. Assessment of ovarian and conceptus development allowed the stage of the oestrous cycle for each animal to be identified (Selwood \& Woolley 1991, Ullmann et al. 2003). A polyclonal antisera against GST-CP4, produced in mice, was used to identify uterine CP4 expression and secretion during the: $3 \mathrm{~mm}$ follicle $(n=3), 4 \mathrm{~mm}$ follicle $(n=2)$, cleavage $(n=5)$, unilaminar blastocyst $(n=3)$, bilaminar blastocyst $(n=1)$, trilaminar blastocyst $(n=1)$, embryonic $(n=3)$ and fetal $(n=5)$ stages of development. Paraffin-embedded uterine sections were placed onto Superfrost slides (Menzel-Glaser, Braunschweig, Germany) and incubated for no longer than $24 \mathrm{~h}$ at $45^{\circ} \mathrm{C}$, before being stored at $4{ }^{\circ} \mathrm{C}$, for no longer than 1 month. Tissue sections were deparaffinized in Histolene (Grale Scientific, Ringwood, VIC, Australia), before being rehydrated in a succession of ethanol washes, each with a progressively higher water content. Incubation in a detergent, $1.0 \%$ Triton X-100 (Promega), for $15 \mathrm{~min}$ improved antibody penetration and reduced background staining. Endogenous peroxidase was blocked by incubation with peroxidase-blocking reagent (DAKO, Carpinteria, CA, USA), and endogenous avidin and biotin activities were blocked by incubation with Avidin/ Biotin-Blocking Kit (Zymed, Mt Waverly, VIC, Australia), both used according to the manufacturer's instructions. The HistostainPlus Broad Spectrum 3,3 diaminobenzidine (DAB) kit (Zymed) was used according to the manufacturer's instructions, before the sections were counterstained with haematoxylin, dehydrated and mounted using DPX (BDH Laboratory Supplies, Poole, England) and allowed to dry for a minimum of $48 \mathrm{~h}$ at $45^{\circ} \mathrm{C}$.

The primary antibody incubation was for $16 \mathrm{~h}$ at $4{ }^{\circ} \mathrm{C}$ using a mouse anti-GST-CP4 antisera diluted to 1:750 in PBS containing $1 \%(\mathrm{w} / \mathrm{v})$ BSA and $10 \%(\mathrm{v} / \mathrm{v})$ inactivated goat 

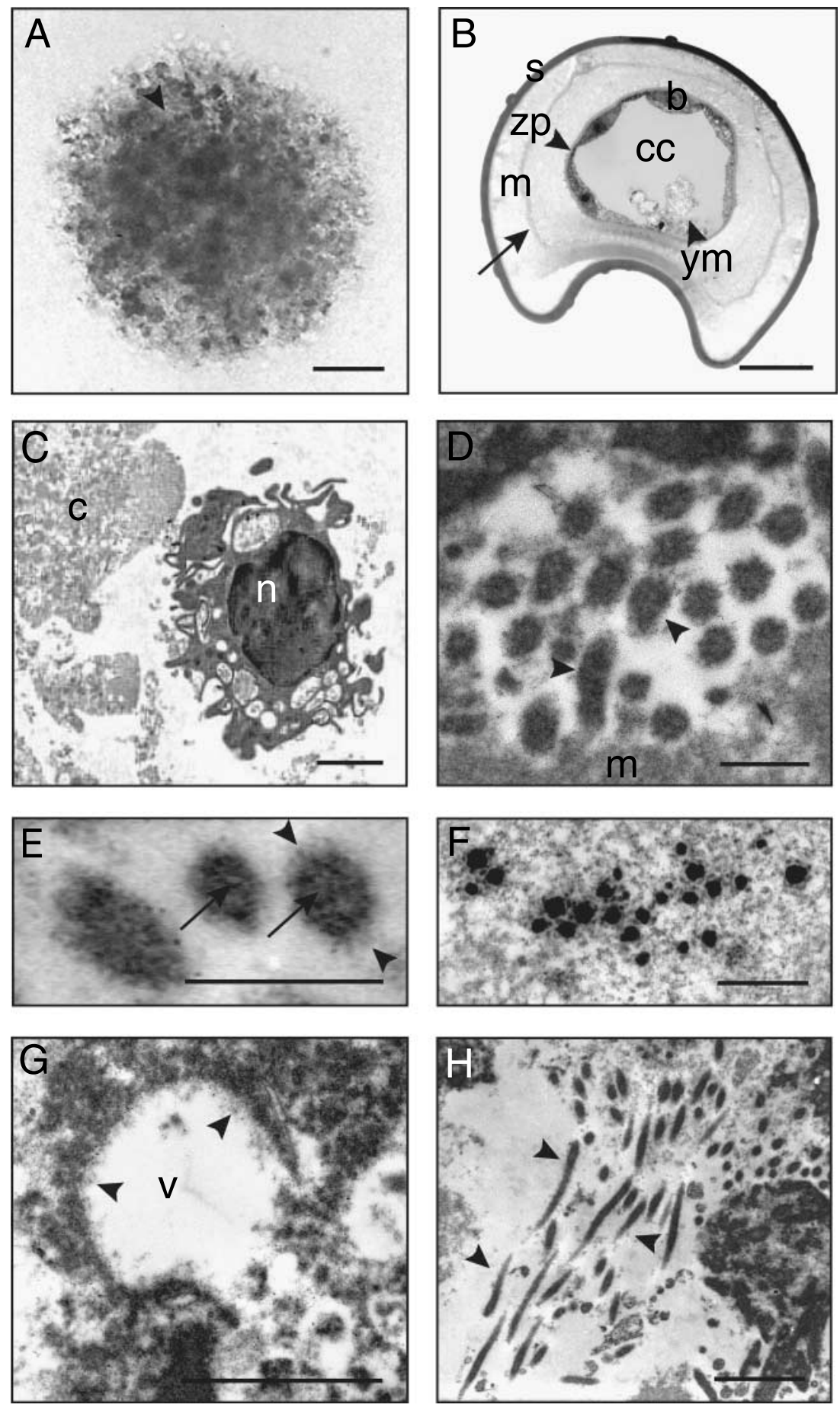

Figure 5 Conceptuses found in the uterine lumen of GST-CP4 immunized female Trichosurus vulpecula: (A) Degraded conceptus, prior to fixation, with no visible shell coat; however, the remains of the mucoid coat were visible (arrowhead). Scale bar represents $100 \mu \mathrm{m}$. (B) Thick section of a 16-cell stage conceptus with apparently normal cells (b), but with irregularities in the mucoid coat $(\mathrm{m})$, specifically the two layers visible (arrow). Scale bar represents $100 \mu \mathrm{m}$. Electron micrographs of: (C) A neutrophil (n) actively phagocytosing the degraded conceptus cytoplasm (c). Scale bar represents $1 \mu \mathrm{m}$. Structures that can be attributed to origin in a conceptus: (D) Sperm tails, indicated by the groupings of flagella (arrowheads) found in the mucoid coat $(\mathrm{m})$. Scale bar represents $1 \mu \mathrm{m}$. (E) Degraded mitochondria with radiating external filaments (arrowheads) and internal cristae (arrows). Scale bar represents $1 \mu \mathrm{m}$. (F) Electrondense granular material in the perivitelline space. Scale bar represents $1 \mu \mathrm{m}$. (G) Degraded vesicles (v) containing electro-lucent granules (arrowheads). Scale bar represents $1 \mu \mathrm{m}$. (H) Microvilli (arrowheads). Scale bar represents $1 \mu \mathrm{m}$. cc, cleavage cavity; m, mucoid coat; $\mathrm{s}$, shell coat; $\mathrm{ym}$, yolk mass; zp, zona pellucida. serum (Zymed). Negative control slides were incubated in 1:750 pre-immune mouse sera, also diluted in PBS containing BSA and inactivated goat serum.

\section{Immunization of $\mathrm{T}$. vulpecula against GST-CP4}

Zoletil (Virbac Animal Health), used at $0.1 \mathrm{mg} / \mathrm{kg}$, sedated animals prior to immunization or blood collection from the lateral or cephalic vein. Blood collection occurred prior to the initial injection, 7-10 days after each injection, then every 3-6 months. Serum was isolated from the blood by centrifugation. Six female and two male $T$. vulpecula were immunized against GST-CP4. One female and two males were immunized against GST, and two males were immunized against PBS, as controls. Four of the seven females used were trapped from the wild, so it was not possible to obtain accurate ages; however, all females were less than 4 years old (according to teeth and condition estimates) and fertile, having successfully 
given birth to a pouch young at least once. Three hundred micrograms of GST-CP4, or GST was emulsified using $0.5 \mathrm{ml}$ Freund's adjuvant (Sigma-Aldrich) and $0.5 \mathrm{ml}$ PBS. Each animal was subcutaneously immunized three times, at 1 -month intervals.

The initial immunization was given in complete Freund's adjuvant, whereas all subsequent immunizations were given in incomplete Freund's adjuvant. A third booster of GST-CP4 was given to three females at 9, 10 or 18 months after the initial immunization.

\section{ELISA}

The immunoplate (Immunolon 4HBX; Thermo LabSystems, Milford, MA, USA) was coated with $0.05 \%$ GST-CP4 diluted in PBS at $4{ }^{\circ} \mathrm{C} \mathrm{O} / \mathrm{N}$ before unoccupied hydrophilic sites were blocked by incubation with 3\% BSA. The sera samples, containing the GST-CP4 antibodies, were serially diluted from 1:200. Protein A (Sigma-Aldrich), conjugated with HRP, was used to detect the antibody, and tetramethyl benzidine (Sigma-Aldrich) was used as the substrate. Absorbance at $450 \mathrm{~nm}$ was read in a microplate reader.

The greatest dilution factor at which the absorbance value of the post-immunization serum sample was higher than the absorbance value of the 1:200 pre-immunization sample, was taken as the end point immune titre for each sample. In most representations of the immune titre, the dilution factor has been converted to its $\log _{2}$ equivalent, so that the values were positive for graphical representation, and to allow statistical analyses (Doolin et al. 2002).

\section{Reproductive monitoring}

Each female was reproductively monitored, using urine sampling as described in Duckworth et al. (1998b), before, during and after immunization against GST-CP4 to identify oestrous cycling. The birth of pouch young (PY) was predicted from reproductive monitoring and confirmed by checking the pouch on the day after the predicted day of birth.

\section{Tissue collection}

Except for two older animals (one male and one female), each animal was killed after a minimum of 12 months post-GST-CP4 immunization. All females were killed during the periovular period, which was identified by reproductive monitoring. Analysis of the female reproductive tract confirmed the stage of the oestrous cycle predicted from reproductive monitoring, and the ipsilateral oviduct and uterus were flushed for the oocyte or conceptus if the ovary had a corpus luteum. The oocyte or conceptus and uteri were fixed and processed for electron microscopy. Non-reproductive somatic tissues (heart, kidney, lens, liver and lung), immune tissues (lymph node and spleen) and reproductive tissues (ovary, oviduct, uterus, epididymis and testis) were collected from animals immunized against GST-CP4, GST and PBS processed for histology and compared with samples from unimmunized animals collected from the wild. Uterine morphology and immune cell distribution was compared between immunized $(n=6)$, control immunized $(n=1)$, unimmunized $(n=17)$ and published standards (Shorey \& Hughes 1973, Padykula 1976, Cruz \& Selwood 1993, 1997, Wick \& Kress 2002).

\section{Statistical analysis}

Fertility was defined as the proportion of oestrous cycles, identified by reproductive monitoring, in which birth of PY occurred. A $\chi^{2}$-test for independence was used to compare fertility (births per oestrous cycle) between the pre- and postimmunization periods. The period of reduced fertility was defined as the period where there were three or fewer PY born in five consecutive oestrous cycles $(<60 \%)$. The relationship between fertility and the immune titre against GST-CP4 during pregnancy was estimated using Pearson's correlations. A onesample $t$-test was used to compare the width of the ipsilateral and contralateral endometrium in GST-CP4 immunized females to the published endometrial width for the periovular period in T. vulpecula: $3 \mathrm{~mm}$ (Shorey \& Hughes 1973). All average values are given as the mean \pm s.E.M.

\section{Declaration of interest}

The authors declare that there is no conflict of interest that could be perceived as prejudicing the impartiality of the research reported.

\section{Funding}

This work was supported by the Foundation for Research Science and Technology (New Zealand) and The University of Melbourne (Australia).

\section{Acknowledgements}

We are grateful for the assistance with animal handling, immunizations and reproductive monitoring provided by Kamani Nanayakkara, Angela Nation, Nicole Griffith and Tania Long, and assistance with histology and electron microscopy from Bruce Abaloz and Joan Clark, all at the Department of Zoology, The University of Melbourne. We also thank Caroline Gargett (Department of Obstetrics and Gynaecology, Monash University) and Lois Salamonsen (Prince Henry's Institute of Medical Research) for their helpful comments on the abnormal uterine morphologies identified.

\section{References}

Casey NP \& Selwood L 2003 Removal of the shell coat affects maintenance of epithelia in blastocysts of the brushtail possum in vitro. Cells, Tissues, Organs 173 21-45.

Casey NP, Martinus R \& Selwood L 2002 Outer egg coats of the marsupial conceptus: secretion and protein composition. Molecular Reproduction and Development 62 181-194.

Croix DA, Samples NK, Vandeberg JL \& Stone WH 1989 Immune response of a marsupial (Monodelphis domestica) to sheep red blood cells. Developmental and Comparative Immunology 13 73-78.

Cruz YP \& Selwood L 1993 Uterine histology of the dasyurid marsupial, Antechinus stuartii: relationship with differentiation of the embryo. Journal of Reproduction and Fertility $99237-242$. 
Cruz YP \& Selwood L 1997 Histological differences between gravid and non-gravid uteri in the dasyurid marsupial, Sminthopsis macroura (Spencer). Journal of Reproduction and Fertility 111 319-325.

Cruz YP, Morton H, Cavanagh AC, Selwood L, Wilson SD \& Sasaki M 2006 Early pregnancy factor in marsupials. Australian Journal of Zoology $\mathbf{5 4}$ 211-215.

Cui S \& Selwood L 2003 Cloning and expression of a novel cDNA encoding shell coat protein, cp4, from the brushtail possum (Trichosurus vulpecula). Molecular Reproduction and Development 65 141-147.

Cui S, Griffith N, Nanayakkara K, Cruz YP \& Selwood L 2001 Developmental targets for fertility control in the brushtail possum. In Report of Conference held under the Auspices of the National Strategy Committee for Possum and Bovine Tb Control, 2-4 April 2001, pp 6-11. Wellington: New Zealand Ministry of Agriculture and Forestry.

Cui S, Nikolovski S, Nanayakkara K \& Selwood L 2005 VAP1, with cystatin c motif, an oocyte protein encoded by a novel ovarian-specific gene during oogenesis in the common brushtail possum (Trichosurus vulpecula). Molecular Reproduction and Development 71 19-28.

Denker H-W \& Gerdes H-J 1979 The dynamic structure of rabbit blastocyst coverings. Anatomica Embryologica 157 15-34.

Doolin E, Wedlock DN, Buddle BM \& Skinner MA 2002 Mucosal immunity in the brushtail possum (Trichosurus vulpecula): detection of antibody in serum and at female reproductive sites after intranasal immunization. Immunology and Cell Biology 80 358-363.

Duckworth JA, Buddle BM \& Scobie S 1998a Fertility of brushtail possums (Trichosurus vulpecula) immunised against sperm. Journal of Reproductive Immunology 37 125-138.

Duckworth JA, Scobie S, Jones DE \& Selwood L 1998b Determination of oestrus and mating in captive female brushtail possums, Trichosurus vulpecula (Marsupialia: Phalangeridae), from urine samples. Australian Journal of Zoology 46 547-555.

Frankenberg S \& Selwood L 1998 An ultrastructural study of the role of an extracellular matrix during normal cleavage in a marsupial, the Brushtail Possum. Molecular Reproduction and Development 50 420-433.

Gockel CM, Bav S \& Beagley KW 2000 Transcutaneous immunization induces mucosal and systemic immunity a potent method for targeting immunity to the female reproductive tract. Molecular Immunology $\mathbf{3 7}$ 537-544.

Herrler A, Stewart F, Crossett B, Pell JM, Ellis PD, Brown KD, Allen WR \& Beier HM 1998 Proteins in the coats of preimplantation rabbit and horse embryos. Zygote 6 (Supplement 1) S47-S48.

Kay DJ \& Kitchener AL 2003 Immune response of the tammar wallaby (Macropus eugenii) to sperm antigens. Reproduction, Fertility, and Development 15 429-437.

Kitchener AL, Edds LM, Molinia FC \& Kay DJ 2002 Porcine zonae pellucidae immunization of tammar wallabies (Macropus eugenii): fertility and immune responses. Reproduction, Fertility, and Development $\mathbf{1 4}$ 215-223.

Lewis GS 2004 Steroidal regulation of uterine immune defences. Animal Reproduction Science 82-83 281-294.

Moore HDM \& Taggart DA 1993 In vitro fertilization and embryo culture in the grey short-tailed opossum Monodelphis domestica. Journal of Reproduction and Fertility 98 267-274.

Morton H 1998 Early pregnancy factor: an extracellular chaperonin 10 homologue. Immunology and Cell Biology 76 483-496.

Padykula HA 1976 Cellular mechanisms involved in cyclic stromal renewal of the uterus. III. Cells of the immune response. Anatomical Record 184 49-72.

Pancholi V 2001 Multifunctional $\alpha$-enolase: its role in diseases. Cellular and Molecular Life Sciences 58 902-920.
Piatigorsky J 1989 Lens crystallins and their genes: diversity and tissuespecific expression. FASEB Journal 3 1933-1940.

Piccinni M-P 2003 Role of immune cells in pregnancy. Autoimmunity 36 $1-4$.

Quayle AJ 2002 The innate and early immune response to pathogen challenge in the female genital tract and the pivotal role of epithelial cells. Journal of Reproductive Immunology 57 61-79.

Renfree MB \& Lewis AM 1996 Cleavage in vivo and in vitro in the marsupial Macropus eugenii. Reproduction, Fertility, and Development 8 725-742.

Robertson SA 2000 Control of the immunological environment of the uterus. Reviews of Reproduction 5 164-174.

Rowlands DT Jr 1970 The immune response of adult opossums (Didelphis virginiana) to the Bacteriophage f2. Immunology 18 149-155.

Selwood L 2000 Marsupial egg and embryo coats. Cells, Tissues, Organs 166 208-216.

Selwood L \& Woolley PA 1991 A timetable of embryonic development, and ovarian and uterine changes during pregnancy, in the stripe-faced dunnart, Sminthopsis macroura (Marsupialia: Dasyuridae). Journal of Reproduction and Fertility 91 213-227.

Shorey CD \& Hughes RL 1973 Cyclical changes in the uterine endometrium and peripheral plasma concentrations of progesterone in the marsupial Trichosurus vulpecula. Australian Journal of Zoology 21 1-19.

Targowski SP 1984 Immune-mediated migration of neutrophils into the uterine lumen of guinea pigs. American Journal of Reproductive Immunology 6 190-194.

Taylor DL \& Burrell R 1968 The immunologic responses of the North American opossum (Didelphis virginiana). Journal of Immunology 101 $1207-1216$.

Ullmann SL, Russell AJ, Mason JI \& Selwood L 2003 Species differences in the ovarian distribution of $3 \beta$-hydroxysteroid dehydrogenase $/ \Delta^{5-4}$ isomerase ( $3 \beta-\mathrm{HSD})$ in two marsupials: the brushtail possum Trichosurus vulpecula and the grey, short-tailed opossum Monodelphis domestica. Reproduction 125 65-73.

Wick R \& Kress A 2002 Ultrastructural changes in the uterine luminal and glandular epithelium during the oestrous cycle of the marsupial Monodelphis domestica (Grey Short-Tailed Opossum). Cells, Tissues, Organs 170 111-131.

Wilkinson R, Kotlarski I \& Barton M 1994 Further characterisation of the immune response of the koala. Veterinary Immunology and Immunopathology 40 325-339.

Wira CR \& Rossoll RM 1995 Antigen-presenting cells in the female reproductive tract: influence of the estrous cycle on antigen presentation by uterine epithelial and stromal cells. Endocrinology 136 4526-4534.

Wistow G \& Kim H 1991 Lens protein expression in mammals: taxonspecificity and the recruitment of crystallins. Journal of Molecular Evolution 32 262-269.

Wistow GJ \& Piatigorsky J 1988 Lens crystallins: the evolution and expression of proteins for a highly specialized tissue. Annual Reviews in Biochemistry 57 479-504.

Wistow GJ, Lietman T, Williams LA, Staple SP, de Jong WW, Horwitz J \& Piatigorsky J $1988 \tau$-Crystallin/ $\alpha$-enolase: one gene encodes both an enzyme and a lens structural protein. Journal of Cellular Biology 107 $2729-2736$.

Received 28 September 2007

First decision 20 November 2007

Revised manuscript received 19 June 2008

Accepted 8 July 2008 